\title{
Racial variations in response to intralesional collagenase clostridium histolyticum in men with Peyronie's disease
}

\author{
Hoang Minh Tue Nguyen, Kenneth J. DeLay, Linley Diao, Nora M. Haney, James Anaissie, Faysal A. Yafi, \\ Suresh C. Sikka, Wayne J. G. Hellstrom \\ Department of Urology, Tulane University School of Medicine, New Orleans, LA, USA \\ Contributions: (I) Conception and design: HM Nguyen, J Anaissie; (II) Administrative support: SC Sikka, WJ Hellstrom, KJ DeLay; (III) Provision \\ of study materials or patients: SC Sikka, WJ Hellstrom, KJ DeLay; (IV) Collection and assembly of data: HM Nguyen, L Diao, KJ DeLay; (V) Data \\ analysis and interpretation: HM Nguyen, J Anaissie; (VI) Manuscript writing: All authors; (VII) Final approval of manuscript: All authors. \\ Correspondence to: Wayne J. G. Hellstrom, MD. Department of Urology, Tulane University Health Sciences Center, 1430 Tulane Ave. 86-42, New \\ Orleans, LA 70112, USA. Email: whellst@tulane.edu.
}

\begin{abstract}
Background: An examination of potential racial variations between Caucasian American (CA) and African American (AA) men in the efficacy and safety of collagenase clostridium histolyticum $(\mathrm{CCH})$ in the treatment of Peyronie's disease (PD).

Methods: We retrospectively reviewed the records for all patients treated with CCH for PD between 04/2014 and 05/2017. Patients were divided into two groups based on their self-reported race. The primary outcomes of interest were final change in curvature after $\mathrm{CCH}$ treatment regardless of number of $\mathrm{CCH}$ cycles received, and frequency of treatment-related adverse events (TRAEs).

Results: A total of 159 patients were included in the study, of which 146 (91.8\%) were CA while $13(8.2 \%)$ were AA. Mean duration of PD was 28.3 months for CA patients and 16.8 months for CA patients $(\mathrm{P}=0.436)$. There was no significant difference in final change in curvature CA and AA patients $\left(15.9^{\circ} v s\right.$. $13.1^{\circ}$ respectively, $\left.\mathrm{P}=0.445\right)$. There was no statistically significant difference in frequency of TRAEs between CA patients (17, 12\%) and AA patients $(0,0 \%)(\mathrm{P}=0.208)$.

Conclusions: Although racial variations in efficacy and safety of $\mathrm{CCH}$ in patients with $\mathrm{PD}$ have not been addressed in the literature, preliminary results from our study suggest that $\mathrm{CCH}$ therapy may be equally efficacious and safe in both CA and AA men. There was no statistically significant difference in final change in curvature, International Index of Erectile Function (IIEF) scores, penile length, or TRAEs after CCH treatment between $\mathrm{CA}$ and AA patients.
\end{abstract}

Keywords: Collagenase clostridium histolyticum (CCH); erectile function; Peyronie's disease (PD); racial variations

Submitted Jun 27, 2017. Accepted for publication Jul 17, 2017.

doi: 10.21037/tau.2017.07.26

View this article at: http://dx.doi.org/10.21037/tau.2017.07.26

\section{Introduction}

Peyronie's disease (PD), defined as the abnormal formation of fibrous plaques in the tunica albuginea of the penis, is a chronic condition that can lead to severe physical deformity and psychological distress in affected patients (1). PD is strongly associated with comorbid erectile dysfunction (ED), which often leads to depression and relationship difficulties $(2-5)$. PD most commonly presents in men in their 50 s with penile deformity, sometimes with pain. These severe physical and emotional burdens make it crucial to fully understand PD.

PD afflicts about $4 \%$ of the U.S. adult male population, with a much higher reported prevalence in Caucasian American (CA) than other races (6). In a population-based study by DiBenedetti et al., $75.2 \%$ of patients with PD were CA while only $9.9 \%$ of the PD patients were African American (AA) (6). A retrospective review of data from 
hospitals in New Orleans from 1994 to 2000 by Shaw et al. found that $77.6 \%$ of $\mathrm{PD}$ patients were CA, and $19.4 \%$ were AA (7). Collagenase Clostridium histolyticum (CCH, $\mathrm{Xiaflex}^{\circledR}$, Auxilium, Chesterbrook, PA) is an injectable agent that enzymatically degrades the interstitial collagen in PD plaques, and was shown to reduce penile curvature by an average of $35 \%$ in the IMPRESS (Investigation of Maximal Peyronie's Reduction Efficacy and Safety Studies) trials (8). These trials recommended that each treatment cycle consist of two intralesionally injected doses of $\mathrm{CCH}$, separated by 24-72 hours, into the penile plaque causing the curvature. Treatment cycles are repeated every 6 weeks for up to four treatment cycles.

Although there exists a plethora of literature describing the safety and efficacy of $\mathrm{CCH}$ in the treatment of $\mathrm{PD}$, racial variation between $\mathrm{CA}$ and $\mathrm{AA}$ men who undergo $\mathrm{CCH}$ treatment remains to be elucidated. The aim of this study is to determine if there is a racial variation between $\mathrm{CA}$ and $\mathrm{AA}$ in outcome after $\mathrm{CCH}$ treatment.

\section{Methods}

\section{Patient population}

Retrospective data were collected for consecutive patients with PD who underwent treatment with $\mathrm{CCH}$ between April 2014 and May 2017 at one institution. A total of 159 patients were included in the study. Race was self-reported by patients. Patients with ventral curvature, hourglass deformity, initial curvature $<30^{\circ}$, and calcified penile plaques were excluded. Three patients of races other than CA and AA were also excluded. The medical records were reviewed and data were collected pre- and post-treatment. Variables of interest included demographics, penile curvature measurements, penile vascular findings, sexual function measured via International Index of Erectile Function (IIEF) scores, flaccid, stretched and erect penile length, and other treatment outcomes including complications and the need for secondary procedures. Our institutional review board approved the study.

\section{Efficacy and safety endpoints}

The primary outcome of interest was the final change in curvature after finishing $\mathrm{CCH}$ therapy, regardless of number of cycles received. Secondary efficacy outcomes of interest included overall change in IIEF score, and change in curvature after the first cycle. The primary endpoint used to evaluate safety was the frequency of serious treatment- related adverse event (TRAE), defined as a complication that occurred during $\mathrm{CCH}$ treatment that the administering physician considered to be directly caused by $\mathrm{CCH}$ therapy. These included corporal rupture, penile hematoma, swelling, and hematuria following $\mathrm{CCH}$ injection.

\section{Intralesional injections of $\mathrm{CCH}$}

Patients qualified for $\mathrm{CCH}$ therapy if they had PD with a palpable, non-calcified penile plaque and a non-ventral curvature deformity of at least $30^{\circ}$ on initial duplex measurement. At each first visit per cycle, an erection was induced by intracavernosal injection (ICI) of alprostadil (6-20 $\mu \mathrm{g})$. The area of maximal curvature was then marked for injection. The dose of $\mathrm{CCH}$ used was 10,000 biofactor units $(\mathrm{ABU})$ per injection, which equates to $0.58 \mathrm{mg}$. Each treatment cycle consisted of two intralesional injections of $\mathrm{CCH}$, separated by 24-72 hours, administered while the penis was on stretch in the flaccid state. Treatment cycles were repeated every 6 weeks, for up to four cycles. Penile modeling was initiated 24-72 hours by the patient following the second injection of each treatment cycle.

\section{Statistical analysis}

Statistical analyses were performed using the STATA statistical software package version 12.1 (StataCorp LLC, College Station, TX, USA). Continuous data are represented by means and the standard deviation (SD). Twotailed Student's $t$-test was used for intergroup comparisons for continuous variables and chi square tests for categorical variables. A $\mathrm{P}<0.05$ was considered statistically significant.

\section{Results}

\section{Pre-treatment characteristics}

Of the 159 patients included in the study, 146 (91.8\%) were CA while 13 (8.2\%) were AA. Mean duration of PD was 28.3 months for CA patients and 16.8 months for AA patients $(\mathrm{P}=0.436)$. There was no statistically significant difference between CA and AA men in mean pre-treatment curvature $\left(57.7^{\circ}\right.$ vs. $61.2^{\circ}$ respectively, $\left.\mathrm{P}=0.454\right)$ or pretreatment IIEF scores (17.8 vs. 16.6 respectively, $\mathrm{P}=0.476$ ). There are statistically significant differences between CA and AA men in pre-treatment flaccid penile length $(9.2 \mathrm{vs}$. $10.5 \mathrm{~cm}$ respectively, $\mathrm{P}=0.003$ ) and pre-treatment stretched penile length (14.0 vs. $15.2 \mathrm{~cm}$ respectively, $\mathrm{P}=0.037)$. In 
terms of comorbidities, the only statistically significant difference between CA and AA patients are in prevalence of diabetes (14.4 vs. $46.2 \%, \mathrm{P}=0.003)$. The mean number of cycles for all patients was 3.2 (SD 1.2), with $13.8 \%$ completing one cycle, $12.6 \%$ two cycles, $20.1 \%$ three cycles, $50.9 \%$ four cycles, and $2.5 \%>$ four cycles, with no statistically significant difference observed between the two groups (3.2 vs. 2.8 cycles, $\mathrm{P}=0.283$ ). Most patients in our study were still undergoing treatment at the time of data collection. Reasons for treatment discontinuation before the completion of four cycles included lack of response, traveling from long distance, complications from prior injections, lack of insurance coverage, and significant improvement in curvature. All other pre-treatment characteristics are summarized in Table 1.

\section{Efficacy outcomes}

For all patients, regardless of PD phase or number of $\mathrm{CCH}$ cycles received, curvature improved from 58 degrees pre-treatment to 42.3 degrees post-treatment $(\mathrm{P}<0.001)$. Between CA and AA patients, there was no statistically significant difference in final change in curvature $\left(15.9^{\circ}\right.$ vs. $13.1^{\circ}$ respectively, $\left.\mathrm{P}=0.445\right)$, even when stratified by $\mathrm{CCH}$ cycle (Figure 1). In addition, there is no statistically significant difference between CA and AA patients in change in stretched penile length $(0.19$ vs. $-0.25 \mathrm{~cm}$ respectively, $\mathrm{P}=0.593$ ) or change in IIEF after treatment (-0.46 vs. $0, \mathrm{P}=0.892)$. Primary and secondary efficacy outcomes are summarized in Table 2.

\section{Safety outcomes}

There was no statistically significant difference in frequency of TRAEs between CA $(17,12 \%)$ and AA patients $(0,0 \%)$ $(\mathrm{P}=0.193)$. Complications of treatment, all in CA patients, included large hematomas in 11 patients, two corporal ruptures, one episode of hematuria following injection, and three episodes of penile swelling. The frequency of TRAEs for each group is listed in Table 3. Assessment was based primarily on history and physical exam. An MRI was used in one patient with equivocal presentation. The two corporal ruptures were treated surgically. Ten (6.3\%) patients required a secondary procedure due to persistent curvature and impaired erectile function. One implantation of an inflatable penile prosthesis was in an AA patient. The other nine surgeries were completed on CA patients and included six penile plications, two implantations of inflatable penile prostheses, and one plaque incision and grafting with no postoperative complications in these ten patients.

\section{Discussion}

To the best of our knowledge, this is the first study comparing the efficacy and safety of CCH therapy between CA and AA patients with $\mathrm{PD}$. Our results suggest that there is no significant difference between $\mathrm{CA}$ and AA patients with $\mathrm{PD}$ in terms of the effectiveness and safety of $\mathrm{CCH}$ use.

In the two large, identical, randomized double-blind, placebo-controlled phase 3 IMPRESS findings, it was shown that $\mathrm{CCH}$ can safely reduce penile curvature by an average of $35 \%$ after four treatment cycles (8). Although these studies were important in demonstrating $\mathrm{CCH}$ efficacy, no analysis was carried out to compare the efficacy of $\mathrm{CCH}$ administration in CA patients $v s$. in AA patients, despite the collection of race as a part of the demographic data. Only $2.9 \%$ of the patients treated in the IMPRESS trials were AA (8). More recent studies involving the efficacy and safety of $\mathrm{CCH}$ therapy investigated other aspects of $\mathrm{CCH}$ therapy, leaving the potential racial variation in $\mathrm{CCH}$ efficacy and safety unclear (9-12).

As the prevalence of PD is much higher among CA men, the studies investigating $\mathrm{CCH}$ therapy in $\mathrm{PD}$ patients are more likely to focus on CA patients than AA patients (6-8). It is important to elucidate whether there is any racial variation between CA and AA patients in effectiveness of $\mathrm{CCH}$ use to determine appropriate practice guidelines. In this retrospective analysis, our results suggest that there is no difference in $\mathrm{CCH}$ treatment efficacy and safety between $\mathrm{CA}$ and AA men. Patients of either race had similar overall changes in penile curvature, IIEF scores, and penile length, indicating that there is no racial variation in $\mathrm{CCH}$ therapy efficacy and safety. These results are reassuring for clinicians and patients who are unsure about the usage of $\mathrm{CCH}$ in AA patients with PD.

Racial variation in the treatment tolerability of PD is another important question that is yet to be answered in the literature. In our analysis, there is no difference in the frequency of TRAEs between CA and AA patients, even though all TRAEs occurred in CA patients. This suggests that $\mathrm{CCH}$ therapy is safe in both $\mathrm{CA}$ and AA patients with PD. These preliminary results are encouraging for AA patients who desire $\mathrm{CCH}$ therapy to treat PD.

The only racial variations in our analysis are diabetes as a comorbidity, flaccid and stretched penile length, and flaccid penile circumference. In our study, AA patients have higher 
Table 1 Pre-treatment characteristics in CA and AA

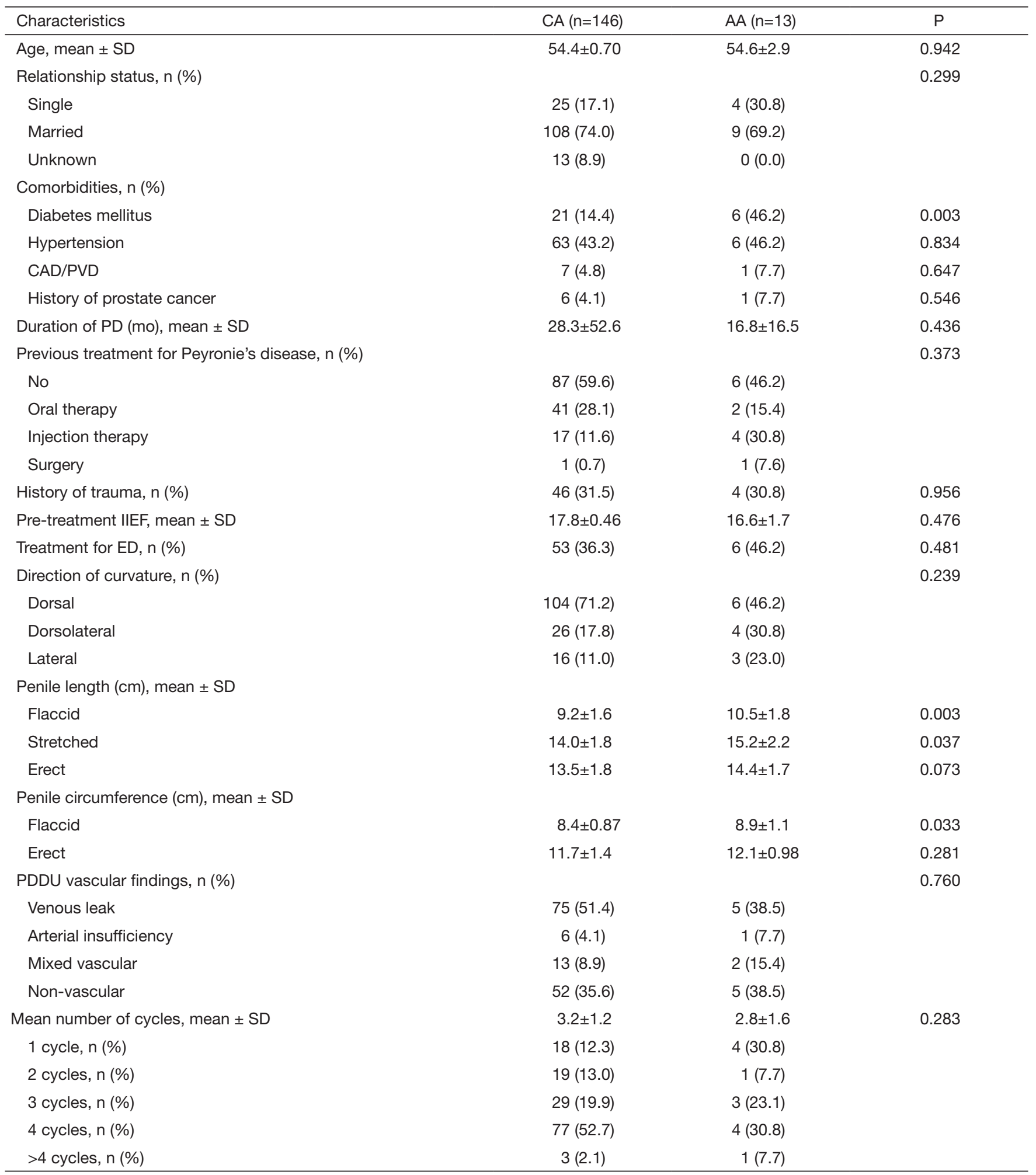

CA, Caucasian American; AA, African American; PD, Peyronie's disease; IIEF, International Index of Erectile Function; ED, erectile dysfunction; SD, standard deviation; CAD, coronary artery disease; PVD, peripheral artery disease. 


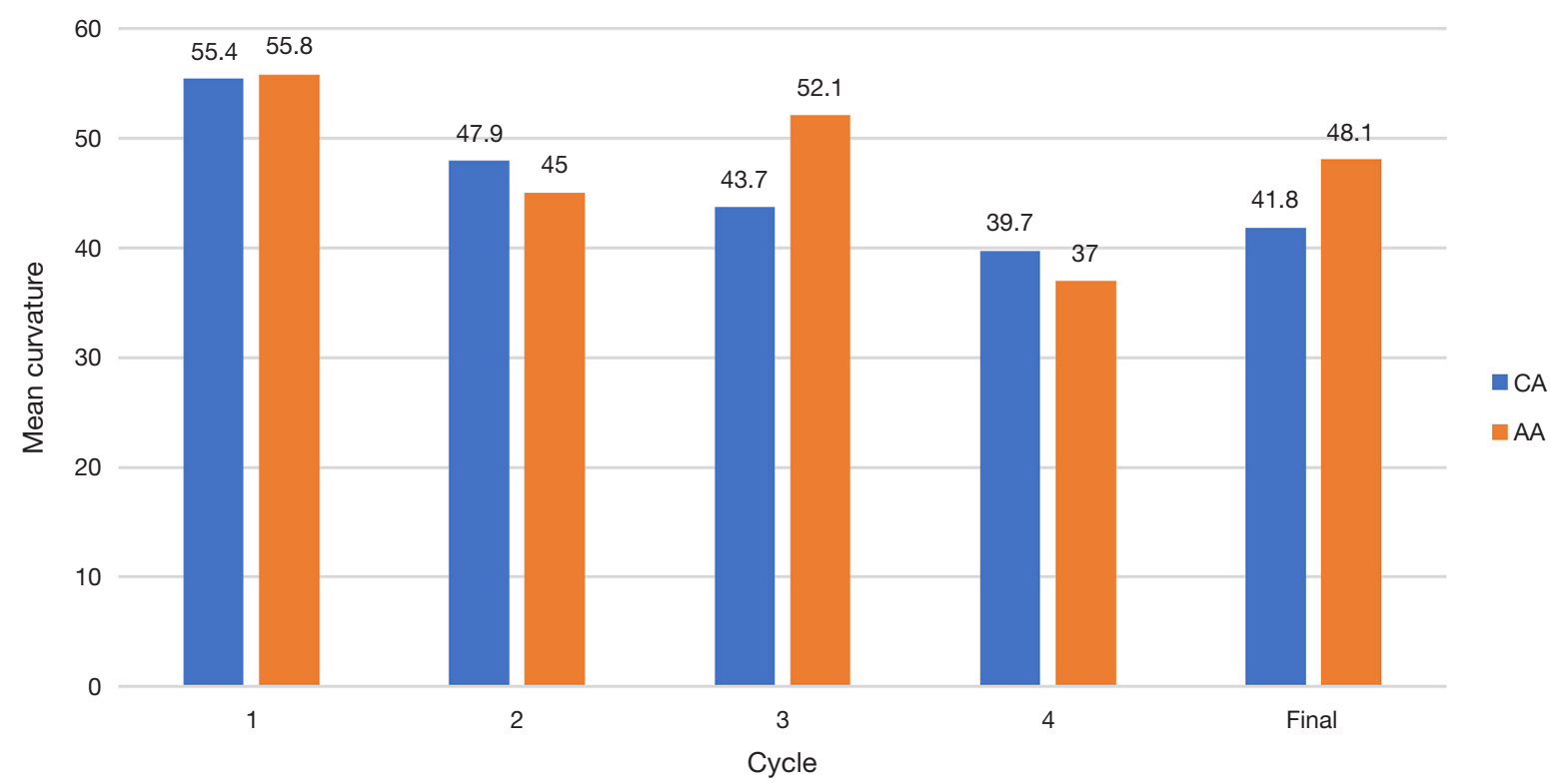

Figure 1 Mean penile curvature at the beginning of each cycle of $\mathrm{CCH}$ therapy, and final curvature for all patients who have received $\mathrm{CCH}$ therapy. Mean change in curvature between each cycle, in order of progressing cycles, is as follows: CA: $-7.5^{\circ},-4.2^{\circ},-4^{\circ}, 2.1^{\circ}$; AA: $-10.8^{\circ}$, $7.1^{\circ},-15.1^{\circ}, 11.1^{\circ}$. None of these changes were statistically significant when comparing between CA and AA. CCH, collagenase clostridium histolyticum; CA, Caucasian American; AA, African American.

Table 2 Efficacy of $\mathrm{CCH}$ on clinical outcomes in CA and AA

\begin{tabular}{lccc}
\hline Outcomes & CA, mean \pm SD & AA, mean \pm SD & P \\
\hline Pre-treatment curvature & $57.7^{\circ} \pm 16.1^{\circ}$ & $61.2^{\circ} \pm 12.1^{\circ}$ & 0.454 \\
Post-treatment curvature & $41.8^{\circ} \pm 15.3^{\circ}$ & $48.1^{\circ} \pm 14.4^{\circ}$ & 0.156 \\
Overall change in curvature & $15.9^{\circ} \pm 12.9^{\circ}$ & $13.1^{\circ} \pm 10.9^{\circ}$ & 0.445 \\
Change in curvature after 1st cycle & $9.3^{\circ} \pm 10.4^{\circ}$ & $9.4^{\circ} \pm 11.3^{\circ}$ & 0.968 \\
Change in IIEF score & $-0.46 \pm 4.7$ & $0 \pm 4.2$ & 0.892 \\
Change in stretched penile length (cm) & $0.19 \pm 1.1$ & $-0.25 \pm 0.35$ & 0.593 \\
\hline
\end{tabular}

$\mathrm{CCH}$, collagenase clostridium histolyticum; CA, Caucasian American; AA, African American; SD, standard deviation; IIEF, International Index of Erectile Function.

Table 3 Frequency of TRAEs for CCH in CA and AA

\begin{tabular}{lccc}
\hline TRAEs & CA (\%) & AA (\%) & P \\
\hline None & $130(89.0)$ & $13(100.0)$ & 0.193 \\
Hematoma & $11(7.5)$ & $0(0.0)$ & \\
Corporal rupture & $2(1.4)$ & $0(0.0)$ & \\
Hematuria & $1(0.7)$ & $0(0.0)$ & \\
Swelling & $3(2.1)$ & $0(0.0)$ & \\
\hline
\end{tabular}

TRAEs, treatment-related adverse events; $\mathrm{CCH}$, collagenase clostridium histolyticum; CA, Caucasian American; AA, African American. prevalence of diabetes, longer flaccid and stretched penile length, and flaccid penile circumference. As diabetes are more prevalent in $\mathrm{AA}$ men with $\mathrm{PD}$, further investigations should be carried out to study if there is a link between PD and diabetes only in AA men. The flaccid and stretched penile length and flaccid penile circumference of both AA and CA patients are within the normal ranges for these measurements (13).

The limitations of this study include bias associated with retrospective studies, a small sample size, and a singlecenter setting. Particularly, we only have 13 AA patients 
vs. $146 \mathrm{CA}$ patients. Another limitation is the variation in number of cycles received. Slightly more than half of our patients completed all four cycles of treatment. The remaining patients either discontinued therapy prematurely or are still continuing therapy. This definitely affects the mean change in curvature, as this calculation was based on final curvature regardless of number cycles received. A last limiting factor was that the Peyronie's disease questionnaire (PDQ), the only currently available validated questionnaire for PD, was not used to assess patient-reported outcomes. Multi-center, randomized-controlled trials with large sample sizes are needed to further analyze the associations reported in this analysis, and further assess potential racial variation in patients with $\mathrm{PD}$.

\section{Conclusions}

Although racial variations in efficacy and safety of $\mathrm{CCH}$ in patients with PD have not been addressed in the literature, preliminary results from our study suggest that $\mathrm{CCH}$ therapy are equally efficacious and safe in both CA and AA men. There was no statistically significant difference in final change in curvature, IIEF, penile length, or TRAEs after CCH treatment between CA and AA patients. Further studies are required to confirm these results.

\section{Acknowledgements}

None.

\section{Footnote}

Conflicts of Interest: WJ Hellstrom is on the speaker's bureau for Endo Pharmaceuticals. He was a principal investigator in the IMPRESS trials. The other authors have no conflicts of interest to declare.

Ethical Statement: The study was approved by The Tulane Biomedical IRB on July 23, 2014 (ID: 630398).

\section{References}

1. Anaissie J, Powers MK, Hellstrom WJ, et al. Collagenase Clostridium histolyticum for the pharmacological management of Peyronie's disease. Drugs Today (Barc) 2015;51:457-68.

2. Gelbard MK, Dorey F, James K. The natural history of Peyronie's disease. J Urol 1990;144:1376-9.
3. Nelson CJ, Diblasio C, Kendirci M, et al. The chronology of depression and distress in men with Peyronie's disease. J Sex Med 2008;5:1985-90.

4. Smith JF, Walsh TJ, Conti SL, et al. Risk factors for emotional and relationship problems in Peyronie's disease. J Sex Med 2008;5:2179-84.

5. Nelson CJ, Mulhall JP. Psychological impact of Peyronie's disease: a review. J Sex Med 2013;10:653-60.

6. Dibenedetti DB, Nguyen D, Zografos L, et al. A Population-Based Study of Peyronie's Disease: Prevalence and Treatment Patterns in the United States. Adv Urol 2011;2011:282503.

7. Shaw K, Puri K, Ruiz-Deya G, et al. Racial considerations in the evaluation of Peyronie's disease. J Urol 2001;165:170:687A.

8. Gelbard M, Goldstein I, Hellstrom WJ, et al. Clinical efficacy, safety and tolerability of collagenase clostridium histolyticum for the treatment of peyronie disease in 2 large double-blind, randomized, placebo controlled phase 3 studies. J Urol 2013;190:199-207.

9. Levine LA, Cuzin B, Mark S, et al. Clinical safety and effectiveness of collagenase clostridium histolyticum injection in patients with Peyronie's disease: a phase 3 open-label study. J Sex Med 2015;12:248-58.

10. Ziegelmann MJ, Viers BR, McAlvany KL, et al. Restoration of Penile Function and Patient Satisfaction with Intralesional Collagenase Clostridium Histolyticum Injection for Peyronie's Disease. J Urol 2016;195:1051-6.

11. Yang KK, Bennett N. Peyronie's Disease and Injectable Collagenase Clostridium histolyticum: Safety, Efficacy, and Improvements in Subjective Symptoms. Urology 2016;94:143-7.

12. Anaissie J, Yafi FA, DeLay KJ, et al. Impact of Number of Cycles of Collagenase Clostridium Histolyticum on Outcomes in Patients With Peyronie's Disease. Urology 2017;100:125-30.

13. Veale D, Miles S, Bramley S, et al. Am I normal? A systematic review and construction of nomograms for flaccid and erect penis length and circumference in up to 15,521 men. BJU Int 2015;115:978-86.

Cite this article as: Nguyen HM, DeLay KJ, Diao L, Haney NM, Anaissie J, Yafi FA, Sikka SC, Hellstrom WJ. Racial variations in response to intralesional collagenase clostridium histolyticum in men with Peyronie's disease. Transl Androl Urol 2017;6(5):888-893. doi: 10.21037/tau.2017.07.26 